\title{
Trans fatty acids, insulin sensitivity and type 2 diabetes
}

\author{
Ulf Risérus
}

Clinical Nutrition and Metabolism, Faculty of Medicine, Uppsala University, Sweden

\begin{abstract}
Trans fatty acids (TFA) could affect cell membrane functions, and may therefore influence peripheral insulin sensitivity and the risk of developing type 2 diabetes. It is important to understand whether low amounts of TFA consumed during long periods may promote insulin resistance and have clinically relevant effects on diabetes risk. Data from controlled intervention studies examining the effects of TFA on insulin sensitivity and type 2 diabetes are reviewed. The results show no consistent effect of TFA on insulin sensitivity in lean healthy subjects, but there is some evidence that TFA could impair insulin sensitivity more than unsaturated fat in subjects with insulin resistance or type 2 diabetes. In particular, conjugated TFA, i.e. certain isomers of conjugated linoleic acid (CLA), impair insulin sensitivity and could promote metabolic disorders. The effect of CLA (trans 10cis 12) on insulin sensitivity and lipid peroxidation is the most dramatic adverse effect described for a dietary fatty acid. CLA isomers are found in relatively low amounts, but long-term exposure may, in theory, have unwanted health effects. The mechanisms of CLA effects are still not completely understood, but may involve increased oxidative stress and inflammation, as well as endothelial dysfunction and direct down-regulating effects on transcription factors required for optimal insulin sensitivity. The inconsistent effect of TFA as a group may be due to methodological limitations (e.g. few studies, with short duration and small sample size) and differences between studies in design, and the type and amount of TFA used. Large controlled trials have been required to demonstrate adverse effects of saturated fat on insulin sensitivity, and similar efforts will be needed to clarify the effect of TFA on insulin sensitivity and diabetes risk. CLA isomers are a group of TFA with potentially adverse effects on glucose metabolism. There are no data to suggest that TFA in general impair insulin sensitivity in practice, compared with such an effect of the much more abundant saturated fatty acids.
\end{abstract}

Keywords: CLA; controlled trial; inflammation; insulin resistance; insulin sensitivity; oxidative stress; trans fatty acids

\section{Introduction}

$\mathrm{E}$ pidemiological data from the Nurses Health Study indicate that trans fatty acids (TFA) increase the risk of developing type 2 diabetes (1). Since insulin resistance is a major contributor to type 2 diabetes, it is of great interest to study the effect of TFA on insulin sensitivity. Insulin resistance (i.e. low insulin sensitivity) can be defined as an impaired tissue response to insulin. Insulin resistance is a central factor in the metabolic syndrome, and is strongly associated with an elevated risk of type 2 diabetes. Insulin resistance and $\beta$-cell dysfunction are major features of type 2 diabetes.

There is fairly convincing evidence from controlled studies to suggest that saturated fatty acids (SFA) impair insulin sensitivity when substituted for unsaturated fats (2). The mechanisms behind such effects are unclear, but could involve several possible components such as altered physiochemical membrane properties, fuel partitioning (via altered fat oxidation and carbohydrate oxidation) or altered gene expression. Recent animal experiments indicate that TFA impair fat cell membrane fluidity and insulin sensitivity, but TFA may also down-regulate peroxisome proliferator-activated receptor- $\gamma$ (PPAR- $\gamma$ ) in adipose tissue (3). The aim of this paper was to review intervention studies that have examined the effects of TFA on insulin sensitivity.

\section{Measurement of insulin sensitivity}

Hyperinsulinaemic euglycaemic clamp is often considered the gold standard to measure insulin sensitivity, whereas the frequently sampled intravenous glucose tolerance test (FSIGT) is a somewhat simpler, yet fairly reliable method. Another way to 
estimate insulin resistance is to measure the insulin response of a glucose tolerance test, which may provide a physiological index of insulin sensitivity. Surrogate markers such as fasting insulin concentrations or the HOMA (homeostatic model assessment) insulin resistance index could also be useful.

\section{Effects of trans fatty acids in healthy subjects}

There are two published studies on healthy subjects. Louheranta et al. (4) studied 14 young healthy Finnish women to investigate the effects of TFA on insulin sensitivity, measured by FSIGT. This was a randomized controlled cross-over study using a diet high in TFA that was compared with a diet high in monounsaturated fatty acids (MUFA). The experimental diets (4 weeks' duration) were compared with a baseline diet [36 energy per cent $(E \%)$ fat, $50 \mathrm{E} \%$ carbohydrates, $15 \mathrm{E} \%$ protein] that the study subjects consumed initially. Thus, the proportion of fat was somewhat higher in comparison with dietary guidelines but reflected a typical diet of the Finnish population. Five energy per cent of the SFA in the baseline diet was replaced by either TFA or MUFA. The diets were designed to retain a stable body weight, and exercise was discouraged during the study. There were no differences between the TFA and MUFA diets in their effects on insulin sensitivity, although fasting insulin concentrations tended to be higher after the TFA diet $(p=0.09)$. There were also no significant differences between diets in the acute insulin response to intravenous glucose. Compliance was good, as indicated by the higher proportions of both elaidic acid (18:1trans) and trans-octadecadienoate (18:2trans) in plasma lipids after the TFA diet compared with the MUFA diet.

Lovejoy et al. (5) performed a study on healthy subjects consisting of a group of 25 American men and women. This randomized cross-over study compared isocaloric diets $(25 E \%$ fat, $57 E \%$ carbohydrates, $15 E \%$ protein) with a 4 week intervention period. A relative dose of $9 E \%$ TFA (18:1trans) was compared with SFA and MUFA. Body weight was kept constant throughout the study. Insulin sensitivity was determined after each diet using FSIGT. Similarly to the previous study (4), there were no differences between the diets in their effects on insulin sensitivity. The SFA diet did, however, impair insulin sensitivity in overweight subjects. In this study, compliance appeared convincing according to serum concentrations of TFA. There was no association between serum levels of 18:1trans and insulin sensitivity.

\section{Effects of trans fatty acids in overweight hyperlipidaemic subjects}

Lichtenstein et al. (6) also investigated the metabolic effect of TFA in overweight, mildly hypercholesterolaemic subjects. In this study insulin sensitivity was not measured directly; instead, fasting insulin levels were measured. Thirty-six middle-aged and older men and women were included in this randomized cross-over study, which had a similar design to the previous studies. Subjects consumed different experimental diets for 5 weeks with a background diet composition of $30 E \%$ fat, $55 E \%$ carbohydrates and $15 E \%$ protein. This was an extensive study as six different diets were compared, including three diets high in TFA $(\sim 3-7 E \%)$. Only data on TFA diets will be briefly reviewed here. Subjects consumed different types of margarine as well as a butter diet, all containing different amounts of TFA. The results showed no dose-response effects of total TFA content on fasting insulin concentrations. However, the butter diet and the diet high in shortening margarine (intermediate 18:2trans content) produced the highest insulin levels among the three TFA diets. The stick margarine diet, which had the highest total TFA content, resulted in lower insulin levels than the other TFA diets. Since insulin sensitivity was not measured directly the results should be interpreted with caution, but they suggest that the type of TFA may be more important than the total amount of TFA.

\section{Effects of trans fatty acids in people with type 2 diabetes}

Christiansen et al. (7) conducted a study on an insulin-resistant group, comprising overweight patients with type 2 diabetes. In this randomized crossover study, isocaloric diets were consumed for 6 weeks, using a high amount of TFA. Twenty energy per cent TFA was compared with $20 E \%$ SFA or $20 E \%$ MUFA, with a background diet containing $30 E \%$ fat, $50 E \%$ carbohydrates and $20 E \%$ protein.

Compared with MUFA, the TFA diet caused a significantly elevated postprandial insulin response to a standardized meal, which was given 6 weeks after the intervention. An elevated insulin response was, however, also observed after SFA, with no significant difference between TFA and SFA. 
A higher insulin response suggests increased insulin resistance, but could also involve impaired insulin clearance or stimulated insulin secretion by $\beta$-cells. It is impossible to conclude whether this reflects an acute postprandial effect of TFA or a chronic effect, since the TFA diet had the same fatty acid composition as the standardized meal.

\section{Conjugated trans fatty acids}

Another type of naturally occurring TFA is conjugated fatty acids. The major form in the diet is conjugated linoleic acid (CLA), which has one of the two double bonds in the trans position. The position of the double bonds varies, resulting in several CLA isomers. Trans 10cis 12-CLA and cis9trans 11-CLA are the two major isomers, the latter being more prevalent in the diet. As cis9trans 11CLA is produced by bacteria in the ruminant gut, CLA is found mainly in dairy products and beef fat. Notably, both CLA isomers are also found in considerable amounts in partially hydrogenated vegetable oils (8). The average intake is about $0.5 \mathrm{~g}$ per day, but this figure varies $(0.1-1.5 \mathrm{~g}$ per day). CLA is also sold in health-food stores as dietary supplements marketed as weight-loss agents and panaceas, based on in vitro and some animal data. In contrast, available clinical data are worrying and suggest several unwanted metabolic effects of CLA. Thus, as there is no clinical benefit of CLA supplements, they should be avoided.

\section{Conjugated linoleic acid and insulin sensitivity}

To investigate the effects of CLA on insulin sensitivity, a double-blinded, randomized controlled trial was carried out with three parallel groups of insulin-resistant men (9). Sixty participants were randomized to capsules containing $3.4 \mathrm{~g}$ of oil $(\sim 1 E \%)$, as a CLA mixture (50:50 mixture with cis 9trans 11-CLA and trans 10cis 12-CLA), a purified trans 10cis 12-CLA preparation or placebo (olive oil). The duration of the study was 3 months, and insulin sensitivity was measured directly by euglycaemic clamp before and after supplementation. Trans 10cis 12-CLA, but not the CLA mixture, impaired insulin sensitivity compared with placebo. Thus, trans 10cis 12-CLA increased insulin resistance as these subjects were already insulin resistant at baseline. In addition, trans 10cis 12-CLA increased fasting glucose concentrations and decreased high-density lipoprotein (HDL)-cholesterol. The CLA mixture also decreased HDL-cholesterol compared with placebo. Furthermore, trans 10cis 12CLA and the CLA mixture increased markers of oxidative stress (urinary $\mathrm{F}_{2}$-isoprostanes) severalfold (10). The former isomer also doubled the plasma concentrations of the inflammatory marker C-reactive protein. Increased insulin resistance was closely and independently correlated with increased oxidative stress (10).

To investigate whether the adverse effect of trans 10 cis 12 -CLA on insulin sensitivity may be an isomer-specific effect, a controlled study was conducted using a purified cis 9trans 11-CLA preparation (11). Compared with trans 10cis 12-CLA, this isomer is more abundant in foods, especially in dairy fats. The design and population were similar to the previous study (9), i.e. a double-blinded controlled study on abdominally obese and insulin-resistant men. The cis9trans11-CLA isomer impaired insulin sensitivity compared with placebo (11). Both studies accord with a recent randomized controlled study in 32 subjects with type 2 diabetes. In that study a CLA mixture impaired both fasting and postprandial insulin sensitivity compared with placebo (12).

\section{Mechanisms of conjugated linoleic acid-induced insulin resistance?}

As mentioned above, oxidative stress and inflammation could be involved in promoting insulin resistance in humans supplemented with high doses of CLA TFA isomers. A recent study in mice supported such a hypothesis, demonstrating increased insulin resistance and signs of local inflammation in adipose tissue, as well as induction of cytokine gene expression after treatment with trans 10cis 12-CLA (13). Adipocytes exposed to CLA secreted more proinflammatory cytokines than controls, and there was an elevated increased macrophage infiltration in adipose tissue treated with trans 10cis 12-CLA (13). These results fit well with the clinical data in prediabetic men (10). Oxidative stress may also impair endothelial function which, in turn, may impair insulin sensitivity. Indeed, it was recently shown in a controlled study that endothelial function (measured by flowmediated dilatation) in healthy subjects was significantly impaired by CLA, and this was accompanied by increased lipid peroxidation or oxidative stress (14).

Another mechanism, as indicated by many studies in CLA-fed mice, is down-regulation of 
PPAR- $\gamma$ in adipose tissue $(13,15,16)$, resulting in subsequent peripheral insulin resistance in skeletal muscle. Data in mice also suggest that CLA could promote a lipodystrophic state, with redistribution of fat stores, i.e. increased liver fat accumulation and decreased peripheral fat, also consistent with down-regulation of PPAR- $\gamma$ (15). Clinical data accord with such intriguing results, although it is premature to conclude that the trans 10 cis CLA isomer has lipodystrophic effects in humans $(9,14)$.

Whether these potential mechanisms behind CLA-induced insulin resistance are shared among all TFA, including those more common in the human diet, is unknown and needs investigation. Nevertheless, these mechanisms provide new insights into the understanding of fatty acid-induced insulin resistance and the metabolic syndrome.

\section{Conclusions}

There are several points and questions that should be considered when interpreting the data published so far on the health effects of TFA. (i) Only shortterm data with varying duration are available so far. (ii) Do the studies have sufficient power? (iii) What is the role of the background diet (high fat versus low fat)? (iv) Are there differences between types (sources) of TFA? (v) Do lean and obese subjects respond differently? (vi) What is the importance of the various amounts of TFA (absolute/relative) used? and (vii) Few studies have used the goldstandard method for determining insulin sensitivity.

The literature suggests that TFA have no significant effect on insulin sensitivity in lean, healthy subjects. However, an elevated insulin response after high TFA intake compared with MUFA in subjects with type 2 diabetes could indicate increased insulin resistance. There are no data to suggest that TFA impair insulin sensitivity compared with SFA, the intake of which is now about 10 times higher than the TFA intake in the Nordic countries. More data are needed before drawing any firm conclusions on the effect of TFA on insulin sensitivity. Furthermore, certain trans CLA isomers (trans10cis 12) impair insulin sensitivity in insulin-resistant and diabetic subjects. The dietary content of CLA is low, but whether dietary exposure to small amounts over long periods could impair insulin action and/or increase diabetes risk is a possibility that requires further investigation. Dietary supplements containing CLA isomers should be discouraged since they have no proven clinical benefit, and may instead be diabetogenic and proatherogenic in insulin-resistant subjects.

\section{Acknowledgements}

The author is supported by the Swedish Society for Medical Research (SSMF) and the Wallenberg Consortium North. Professor Bengt Vessby is acknowledged for his important role in several of the studies presented in this review.

\section{References}

1. Salmeron J, Hu FB, Manson JE, Stampfer MJ, Colditz GA, Rimm EB, et al. Dietary fat intake and risk of type 2 diabetes in women. Am J Clin Nutr 2001; 73: 1019-26.

2. Vessby B, Unsitupa M, Hermansen K, Riccardi G, Rivellese AA, Tapsell LC, et al. KANWU Study. Substituting dietary saturated for monounsaturated fat impairs insulin sensitivity in healthy men and women: the KANWU Study. Diabetologia 2001; 44: 312-9.

3. Saravanan N, Haseeb A, Ehtesham NZ, Ghafoorunissa SA. Differential effects of dietary saturated and trans fatty acids on expression of genes associated with insulin sensitivity in rat adipose tissue. Eur J Endocrinol 2005; 153: $159-65$.

4. Louheranta AM, Turpeinen AK, Vidgren HM, Schwab US, Uusitupa MI. A high-trans fatty acid diet and insulin sensitivity in young healthy women. Metabolism 1999; 48: 870-5.

5. Lovejoy JC, Smith SR, Champagne CM, Most MM, Lefevre M, DeLany JP, et al. Effects of diets enriched in saturated (palmitic), monounsaturated (oleic), or trans (elaidic) fatty acids on insulin sensitivity and substrate oxidation in healthy adults. Diabetes Care 2002; 25: $1283-8$

6. Lichtenstein AH, Erkkila AT, Lamarche B, Schwab US, Jalbert SM, Ausman LM. Influence of hydrogenated fat and butter on CVD risk factors: remnant-like particles, glucose and insulin, blood pressure and C-reactive protein. Atherosclerosis 2003; 171: 97-107.

7. Christiansen E, Schnider S, Palmvig B, Tauber-Lassen E, Pedersen O. Intake of a diet high in trans monounsaturated fatty acids or saturated fatty acids. Effects on postprandial insulinemia and glycemia in obese patients with NIDDM. Diabetes Care 1997; 20: 881-7.

8. Jung MY, Ha YL. Conjugated linoleic acid isomers in partially hydrogenated soybean oil obtained during nonselective and selective hydrogenation processes. J Agric Food Chem 1999; 47: 704-8.

9. Risérus U, Arner P, Brismar K, Vessby B. Treatment with dietary trans 10 cis 12 conjugated linoleic acid causes isomer-specific insulin resistance in obese men with the metabolic syndrome. Diabetes Care 2002; 25: 1516-21.

10. Risérus U, Jovinge S, Basu S, Nordin Fredrikson G, Ärnlöv J, Vessby B. Supplementation with conjugated linoleic acid causes isomer-dependent oxidative stress and elevated C-reactive protein: a potential link to fatty acid induced insulin resistance. Circulation 2002; 106: 1925-9. 
11. Risérus U, Vessby B, Ärnlöv J, Basu S. Effects of cis 9 trans 11 conjugated linoleic acid supplementation on insulin sensitivity, lipid peroxidation and proinflammatory markers in obese men. Am J Clin Nutr 2004; 80: 279-83.

12. Moloney F, Yeow TP, Mullen A, Nolan JJ, Roche HM. Conjugated linoleic acid supplementation, insulin sensitivity, and lipoprotein metabolism in patients with type 2 diabetes mellitus. Am J Clin Nutr 2004; 80: 887-95.

13. Poirier H, Shapiro JS, Kim RJ, Lazar MA. Nutritional supplementation with trans-10,cis-12-conjugated linoleic acid induces inflammation of white adipose tissue. Diabetes 2006; 55: 1634-41.

14. Taylor JS, Williams SR, Rhys R, James P, Frenneaux MP. Conjugated linoleic acid impairs endothelial function. Arterioscler Thromb Vasc Biol 2006; 26: 307-12.

15. Granlund L, Juvet LK, Pedersen JI, Nebb HI. Trans 10,cis 12-conjugated linoleic acid prevents triacylglycerol accumulation in adipocytes by acting as a PPAR $\gamma$ modulator. J Lipid Res 003;44:1441-52.

16. Tsuboyama-Kasaoka N, Takahashi M, Tanemura K, Kim HJ, Tange $\mathrm{T}$, Okuyama $\mathrm{H}$, et al. Conjugated linoleic acid supplementation reduces adipose tissue by apoptosis and develops lipodystrophy in mice. Diabetes 2000; 49: $1534-42$.

\section{Dr Ulf Risérus}

Clinical Nutrition and Metabolism

Department of Public Health and Caring Sciences

Uppsala Science Park

SE-75 I 85 Uppsala

Sweden

Tel: $+46|86| 1797 \mid$

Fax: +46186117976

E-mail: ulf.riserus@pubcare.uu.se 